\title{
The NKI Receptor Antagonist L822429 Reduces Heroin Reinforcement
}

\author{
Estelle Barbier*,', Leandro F Vendruscolo², Joel E Schlosburg ${ }^{2}$, Scott Edwards ${ }^{2}$, Nathan Juergens', \\ Paula E Park ${ }^{2}$, Kaushik K Misra ${ }^{2}$, Kejun Cheng ${ }^{3}$, Kenner C Rice ${ }^{3}$, Jesse Schank', Gery Schulteis ${ }^{4}$, \\ George F Koob ${ }^{2}$ and Markus Heilig'
}

'Laboratory of Clinical and Translational Studies, National Institute on Alcohol Abuse and Alcoholism, Bethesda, MD, USA; ${ }^{2}$ Committee on the Neurobiology of Addictive Disorders, The Scripps Research Institute, La Jolla, CA, USA; ${ }^{3}$ Drug Design and Synthesis Section, Chemical Biology Research Branch, National Institute on Drug Abuse and National Institute on Alcohol Abuse and Alcoholism, National Institutes of Health, Bethesda, MD, USA; ${ }^{4}$ Research Service, VA San Diego Healthcare System, and Department of Anesthesiology, University of California at San Diego School of Medicine, San Diego, CA, USA

\begin{abstract}
Genetic deletion of the neurokinin I receptor (NKIR) has been shown to decrease the reinforcing properties of opioids, but it is unknown whether pharmacological NKIR blockade has the same effect. Here, we examined the effect of L822429, a rat-specific NKIR antagonist, on the reinforcing properties of heroin in rats on short (I h: ShA) or long ( $12 \mathrm{~h}$ : LgA) access to intravenous heroin selfadministration. ShA produces heroin self-administration rates that are stable over time, whereas LgA leads to an escalation of heroin intake thought to model important dependence-related aspects of addiction. L822429 reduced heroin self-administration and the motivation to consume heroin, measured using a progressive-ratio schedule, in both ShA and LgA rats. L822429 also decreased anxietylike behavior in both groups, measured on the elevated plus maze, but did not affect mechanical hypersensitivity observed in LgA rats. Expression of TacRI (the gene encoding NKIR) was decreased in reward- and stress-related brain areas both in ShA and LgA rats compared with heroin-naïve rats, but did not differ between the two heroin-experienced groups. In contrast, passive exposure to heroin produced increases in TacR I expression in the prefrontal cortex and nucleus accumbens. Taken together, these results show that pharmacological NKIR blockade attenuates heroin reinforcement. The observation that animals with ShA and LgA to heroin were similarly affected by $L 822429$ indicates that the SP/NKIR system is not specifically involved in neuroadaptations that underlie escalation resulting from LgA self-administration. Instead, the NKIR antagonist appears to attenuate acute, positively reinforcing properties of heroin and may be useful as an adjunct to relapse prevention in detoxified opioid-dependent subjects.

Neuropsychopharmacology (2013) 38, 976-984; doi:I 0.1038/npp.2012.26I; published online I6 January 2013
\end{abstract}

Keywords: opioids; heroin; self-administration; substance P; neurokinin receptor

\section{INTRODUCTION}

Studies with genetically modified mice have suggested that substance $\mathrm{P}$ (SP) and its preferred neurokinin 1 receptor (NK1R) have a significant role in opioid reinforcement. Morphine reward, as measured by conditioned place preference (CPP), is absent in NK1 $\mathrm{R}^{-1-}$ mice (Murtra et al, 2000), and this is associated with decreased rates of morphine self-administration compared with wild-type mice (Ripley et al, 2002). Neurotoxin lesions targeting NK1 receptor-containing neurons in the amygdala, but not the nucleus accumbens (NAc), prevented the development

* Correspondence: Dr E Barbier, Laboratory of Clinical and Translational Studies, National Institute on Alcohol Abuse and Alcoholism, 10 Center Drive, I0/IE-5330, Bethesda, MD 20892-II08, USA, Tel: +| 30 I 655 4677, Fax: +| 301402 0445, E-mail: barbieres@ mail.nih.gov

Received 8 June 2012; accepted 15 November 2012; accepted article preview online 18 December 2012 of morphine CPP (Gadd et al, 2003), suggesting a role of NK1Rs in the amygdala in mediating opioid reward. A recent study using intracranial self-stimulation (ICSS) has provided further support for a role of NK1Rs in the rewarding effects of opioids, by demonstrating that NK1R blockade attenuated the ability of morphine to lower ICSS thresholds (Robinson et al, 2012). Although these data collectively support the possibility that NK1R antagonists may be useful for the treatment of opioid addiction, demonstration that pharmacological NK1R blockade suppresses self-administration of opioids is lacking.

Initial findings indicated a selective role of NK1Rs in opioid reward and self-administration, in that neither CPP for cocaine (Murtra et al, 2000) nor self-administration of this drug (Ripley et al, 2002) were affected by a genetic deletion of the NK1R. More recently, however, data have also emerged in support of a role for NK1Rs in the rewarding properties of alcohol. For example, NK1 ${ }^{-1-}$ mice consume less alcohol in a two-bottle free-choice 
paradigm, and do not exhibit alcohol CPP (George et al, 2008; Thorsell et al, 2010). The decreased alcohol consumption and reward found in NK1R-null mutants is unlikely to be the result of compensatory developmental processes caused by constitutive gene deletion because these behavioral effects were replicated by NK1R antagonist administration in wildtype animals (Thorsell et al, 2010). Taken together, these observations have provided support for the hypothesis that NK1R antagonism may have utility as a pharmacotherapy for alcoholism. Initial human translation of these findings was obtained through the demonstration that an NK1R antagonist blocked alcohol craving in recently detoxified alcoholdependent patients (George et al, 2008).

Over time, the motivation for drug seeking and taking is hypothesized to undergo an allostatic shift. Positive reinforcement predominates during the early stages of the addictive process, but the ability of drug intake to alleviate negative emotional states that emerge during abstinence becomes increasingly important with a prolonged history of drug abuse (Edwards and Koob, 2010). NK1R antagonists display antistress and antianxiety effects in a wide range of animal models (Ebner and Singewald, 2006), and thereby have the potential to obviate the stressreducing, negatively reinforcing effects of addictive drugs. Accordingly, systemic administration of an NK1R antagonist blocked stress-induced reinstatement of alcohol seeking in rats (Schank et al, 2011). Thus, NK1R antagonist treatment may have a potential for therapeutic utility in addictive disorders through dual mechanisms, by reducing both negative and positive reinforcing properties.

Limited access ( $1 \mathrm{~h}$ per day) to heroin produces stable levels of drug intake. However, extended access (6-23 h per day) to heroin self-administration in rats can lead to escalated self-administration (Ahmed et al, 2000; Vendruscolo et al, 2011). It has been hypothesized that during limited access conditions, drug intake is mainly driven by the positive reinforcing properties of heroin, whereas during extended access, brain stress systems are recruited and negative reinforcement mechanisms predominate (Edwards and Koob, 2010).

Here, we asked whether pharmacological blockade of NK1Rs would be able to reduce heroin reinforcement. Because NK1 receptors display considerable divergence between species, NK1R antagonists developed for activity at the human NK1R may possess limited efficacy in rat studies (Leffler et al, 2009). We therefore used L822429, a brain-penetrant NK1R antagonist with high affinity for the rat NK1R that produces anxiolytic-like effects after systemic administration (Ebner et al, 2004; Ebner et al, 2008; Singewald et al, 2008). We examined the effects of L822429 on heroin self-administration in short (ShA; $1 \mathrm{~h}$ per day) and long access (LgA; $12 \mathrm{~h}$ per day) rats. Furthermore, we tested the effect of NK1R antagonism on measures of negative emotional-like behavior associated with heroin withdrawal: increased anxiety-like behavior and mechanical sensitivity during withdrawal from heroin self-administration in ShA and LgA rats. Finally, to determine neurokinin systemrelated neuroadaptations associated with limited $v s$ excessive heroin intake, TacR1 transcript levels were also assessed in several stress/reward-related brain areas in ShA and LgA rats, and in rats passively receiving repeated moderate doses of heroin or escalating doses of heroin.

\section{MATERIALS AND METHODS}

\section{Subjects}

Adult male Wistar rats (Charles River, Raleigh, NC), weighing between 225 and $275 \mathrm{~g}$ at the beginning of the experiments, were housed in groups of $2-3$ per cage in a temperature-controlled $\left(22^{\circ} \mathrm{C}\right)$ vivarium on a $12 \mathrm{~h} / 12 \mathrm{~h}$ light/dark cycle (lights on at 1800 hours) with ad libitum access to food and water. The animals were allowed to acclimate to the animal facility for at least 7 days before surgery. All procedures adhered to the National Institutes of Health Guide for the Care and Use of Laboratory Animals and were approved by the Institutional Animal Care and Use Committee of The Scripps Research Institute.

\section{Elevated Plus Maze}

The elevated plus maze testing was carried out as described previously (Vendruscolo et al, 2003) during withdrawal, just before subsequent self-administration sessions. The apparatus had four elevated arms ( $52 \mathrm{~cm}$ above the floor), $50 \mathrm{~cm}$ long and $10 \mathrm{~cm}$ wide, set in a cross-like arrangement, with two opposite arms enclosed by $40 \mathrm{~cm}$ high opaque walls and two open arms with a lip ( $1 \mathrm{~mm}$ thick and $5 \mathrm{~mm}$ high). A central platform at their intersection $\left(10 \times 13.5 \mathrm{~cm}^{2}\right)$ permitted access to any of the four arms. The central platform was under $<10 \mathrm{~lx}$ illumination. Each rat was placed on the central platform facing an open arm. The behavior of each animal was recorded for $5 \mathrm{~min}$. The percentage of time spent in the open arms relative to the combined time spent in both the open and closed arms, and the percentage of entries in the open arms were used as indices of anxiety-like behavior. Closed-arm entries were used as an index of general locomotion.

\section{Measurement of Mechanical Sensitivity: Paw Withdrawal Threshold}

Mechanical nociceptive testing was conducted during withdrawal, just before subsequent self-administration sessions according to methods by Chaplan et al (1994) as described previously (Edwards et al, 2012). Briefly, rats were acclimated for $15 \mathrm{~min}$ in elevated cages with a wire mesh floor. A series of von Frey filaments were applied perpendicularly to the plantar surface of the hind paw for $3 \mathrm{~s}$. A sharp withdrawal of the hind paw indicated a positive response. The stimulus was incrementally increased until a positive response was obtained, and then decreased until a negative result was observed to determine a pattern of responses to apply to the statistical method of Dixon (1980). Baseline mechanical nociceptive thresholds were similar to those reported for the ages of rats employed in this study (Ririe and Eisenach, 2006).

\section{Surgery}

Rats were anesthetized with isoflurane (1.5-2.5\%) and prepared with chronic intravenous silastic catheters (Dow Corning, Midland, MI) into the right jugular vein (Vendruscolo et al, 2011). The catheter was secured to the vein with suture thread and passed subcutaneously to exit dorsally on the animal's back. After surgery, the catheters 
were flushed daily with $0.2 \mathrm{ml}$ of a sterile solution containing heparinized (30 USP units $/ \mathrm{ml}$ ) saline and the antibiotic Cefazolin. Rats were allowed to recover for 7 days before behavioral testing.

\section{Self-Administration Procedure}

Self-administration sessions were conducted in standard operant conditioning chambers (Med Associates). Selfadministration sessions were carried out as described previously (Vendruscolo et al, 2012). Briefly, rats were trained to press one of the two levers (the active lever) on a fixed-ratio 1 (FR1) schedule of reinforcement (each response resulted in fluid delivery) to obtain $0.1 \mathrm{ml}$ of heroin $(60 \mu \mathrm{g} / \mathrm{kg}$ per infusion) in 1-h sessions. Reinforced responses were followed by a 20-s timeout period, in which a cue-light (above the active lever) was turned on and lever presses did not result in additional injections. During the acquisition of heroin self-administration, food and water were not available to the rats while in the test chambers. After the acquisition of heroin self-administration, rats were split into two groups matched for lever press in the last three sessions of the acquisition phase and were given $1 \mathrm{~h}$ (ShA) or $12 \mathrm{~h}(\mathrm{LgA})$ of access to heroin self-administration. In this phase, all groups were allowed to nosepoke for food on an FR3 schedule and water on an FR1 schedule while in the test chambers. (Vendruscolo et al, 2011).

\section{Pharmacological Testing}

The NK1 receptor antagonist L822429 was prepared as a suspension for systemic administration (intraperitoneally) in $45 \%(\mathrm{w} / \mathrm{v}) 2$-hydroxypropyl $\beta$-cyclodextrin. Rats were administered vehicle or L822429 (15 and $30 \mathrm{mg} / \mathrm{kg}$ in $45 \%$ (w/v) 2-hydroxypropyl $\beta$-cyclodextrin at $2 \mathrm{ml} / \mathrm{kg}$ ). Doses and pretreatment times were based on previous studies (Schank et al, 2011).

For the self-administration studies, rats were given L822429 $1 \mathrm{~h}$ before session. ShA and LgA rats received all three doses $(0,15$, and $30 \mathrm{mg} / \mathrm{kg})$ in a Latin Square design and tested for heroin self-administration on an FR1 schedule. A regular FR1 heroin self-administration session without drug treatment was performed between testing days. Operant self-administration on FR1 requires minimal effort from the animal to obtain heroin and was considered herein a measure of intake.

After the FR1 treatment phase was completed, the same ShA and LgA rats were then tested on a progressive-ratio (PR) schedule, under which the number of lever presses necessary to obtain the next reinforcement increased progressively according to the following progression: 1,1 , $2,2,2,3,3,4,4,5,5,6,6,7,7,8,9,9,10,11,11,12,13,14$, 14, etc. (Vendruscolo et al, 2012). The PR session lasted $6 \mathrm{~h}$. Rats received 0 and $30 \mathrm{mg} / \mathrm{kg}$ in a Latin Square design (with a regular FR1 session between the PR sessions). In this test, the workload ('price') for the next reinforcement increases progressively and is considered herein a measure of motivation.

Rats from a separate cohort were trained to selfadminister heroin and split in ShA and $\operatorname{LgA}$ groups as described above. When escalated levels of heroin intake were established for the LgA group (ie, significantly escalated intake over 1 week), ShA and LgA rats were given L822429 (0 or $30 \mathrm{mg} / \mathrm{kg}$, intraperitoneally, $1 \mathrm{~h}$ before testing) and tested in the elevated plus maze. Rats were tested only once in this test with a single dose (ie, either 0 or $30 \mathrm{mg} / \mathrm{kg}$ ). The same animals were tested for PWT afterward (ie, $1.5 \mathrm{~h}$ post injection). After 2 days, rats received the other dose (different from the first day) and tested for PWT only. Testing was performed during acute withdrawal (12-23 $\mathrm{h}$ post heroin self-administration, corresponding to the time of day when ShA and LgA animals would typically be selfadministering heroin).

\section{Passive Heroin Administration}

To determine the effects of passively administered heroin on gene expression, we chronically treated rats with heroin for 18 days, either at a constant level (repeated heroin group: $1.25 \mathrm{mg} / \mathrm{kg}$ subcutaneously, days $1-18$ ) or via an escalating dose regimen (escalated heroin group: $1.25 \mathrm{mg} / \mathrm{kg}$ subcutaneously, days $1-3 ; 2.5 \mathrm{mg} / \mathrm{kg}$, days $4-6$; $5 \mathrm{mg} / \mathrm{kg}$, days $7-9 ; 10 \mathrm{mg} / \mathrm{kg}$, days $10-12 ; 20 \mathrm{mg} / \mathrm{kg}$, days $13-$ 18). Animals receiving chronic saline served as a control group (repeated saline group: $1 \mathrm{ml} / \mathrm{kg}$ subcutaneously, days 1-18). All treatments were administered once per day. Paw withdrawal thresholds (PWTs) were measured on day 18 immediately before the final injection, and animals were killed $24 \mathrm{~h}$ following this last injection.

\section{Reverse Transcription and Quantitative PCR}

Prefrontal cortex (PFC), NAc, BNST, amygdala, and hippocampus (Hipp) were collected 20,10 , and $24 \mathrm{~h}$ after ShA self-administration, LgA self-administration, and passive heroin administration, respectively. For self-administration groups, this time point roughly corresponded to the typical withdrawal time between sessions. RNA was extracted and purified from brain tissue using the PureLink RNA Mini Kit (Ambion, Austin, TX) following the manufacturer's instructions. cDNA was reverse transcribed from total RNA using the Superscript III First Strand Synthesis System (Invitrogen, Carlsbad, CA). Gene expression levels were determined by quantitative polymerase chain reaction (qPCR) using a TaqMan Universal PCR Master Mix (Applied Biosystems, Foster City, CA). Reactions were carried out as described previously (Vendruscolo et al, 2012) and cDNA concentrations of TacR1 were calculated according to the relative quantification (ddCt) method, corrected for differences in PCR efficiency, normalized to glyceraldehyde-3-phosphate dehydrogenase (Gapdh). Primers used were as follows: TaqMan qPCR utilized commercially available TacR1 (Rn00562004_ml) and Gapdh (Rn99999916_s1) primer/probe sets (Applied Biosystems), with PCR conditions according to the manufacturer's protocol.

\section{Statistical Analysis}

All data are expressed as means and standard errors of the mean (SEM). Data were analyzed using a one-way analysis of variance (ANOVA) with treatment (repeated saline, repeated heroin, and escalated heroin) as a betweensubjects factor or using a two-way repeated measure 
ANOVA, with group (ShA and $\operatorname{LgA}$ ) as the betweensubjects factor and treatment $(0,15$, and $30 \mathrm{mg} / \mathrm{kg}$ or 0 and $30 \mathrm{mg} / \mathrm{kg}$ ) and session or time as the within-subjects factor. For elevated plus maze, data were analyzed using a two-way ANOVA, with group (ShA and LgA) and treatment ( 0 and $30 \mathrm{mg} / \mathrm{kg}$ ) as the between-subjects factors. Differences in PWTs across tests were analyzed using two-way ANOVA with group (naïve, ShA, and LgA) as the betweensubjects factor and treatment ( 0 and $30 \mathrm{mg} / \mathrm{kg}$ ) as the within-subjects factor. For TacR1 mRNA levels, data were analyzed using a one-way ANOVA with group (naïve, ShA, and LgA; repeated saline, repeated heroin, and escalated heroin) as the between-subjects factor. When appropriate, post hoc comparisons were performed using the Fisher's least significance difference (LSD) test. The probability for a Type 1 error for all significance testing was set at $P \leqslant 0.05$.

\section{RESULTS}

NK1R Antagonism Decreases Heroin SelfAdministration and Motivation to Obtain Heroin in Both ShA and LgA Rats

ShA rats displayed stable levels of heroin self-administration (Figure 1a), whereas LgA rats displayed an escalation of intake (group $\times$ session interaction: $F_{(14,518)}=15.8$, $P<0.0001)$ that became significant by session 5 $(P<0.0001)$. Systemic injection of $30 \mathrm{mg} / \mathrm{kg} \quad \mathrm{L} 822429$ significantly decreased heroin self-administration in the first hour under an FR1 schedule of reinforcement (Figure 1b) in both ShA and LgA rats (treatment effect: $\mathrm{F}_{(2,36)}=7.7, P<0.005$ followed by Fischer's LSD post hoc, $P<0.001)$. The statistical analysis also revealed a time $\times$ treatment interaction $\left(\mathrm{F}_{(8,72)}=2.1, P<0.05\right)$ for cumulative heroin infusions in the entire 12 -h session, with selfadministration significantly decreased by $30 \mathrm{mg} / \mathrm{kg}$ L822429 compared with vehicle $(P<0.005)$ at 6 and $12 \mathrm{~h}$ (Figure $1 \mathrm{c}$ ).

Food intake during the heroin self-administration session was also significantly affected by the treatment $\left(\mathrm{F}_{(2,36)}=11.3, \quad P<0.001\right)$. The highest dose of L822429 significantly decreased food intake by ShA and LgA rats $(P<0.05$; Figure $1 \mathrm{~d})$. However, water intake was unaffected by the treatment, suggesting that the drug did not suppress overall motor behavior (Table 1).

In the PR, LgA rats displayed a greater motivation for heroin, measured by the number of drug infusions earned in the 6-h session, compared with ShA rats (overall group effect: $\left.\mathrm{F}_{(1,18)}=10.0, P<0.005\right)$. In all, $30 \mathrm{mg} / \mathrm{kg} \mathrm{L} 822429$ significantly decreased the motivation for heroin consumption in both ShA and LgA rats compared with vehicle (overall treatment effect: $\mathrm{F}_{(1,18)}=7.9, P<0.05$; Figure 1d). The ANOVA also revealed a treatment $v s$ time interaction $\left(\mathrm{F}_{(5,90)}=7.0, P<0.0001\right)$, indicating that the drug was more effective in reducing responding later on the session when the workload for heroin reinforcement is higher.

\section{NK1R Antagonism Produces an Anxiolytic-Like Effect in ShA and LgA Rats}

Anxiety-like behavior was measured using the elevated plus maze for drug-naïve, ShA, and LgA heroin self-administering groups, shown in Figure 2. Overall group effects were detected for the $\%$ time in the open arms $\left(\mathrm{F}_{(2,55)}=4.0\right.$, $P<0.05)$ and $\%$ entries in the open arms $\left(\mathrm{F}_{(2,55)}=4.6\right.$, $P<0.05)$. LgA rats spent less time in the open arms compared with naïve rats $(P<0.01)$, and both ShA $(P<0.05)$ and $\operatorname{LgA}(P<0.01)$ made fewer entries in the open arms compared with naïve rats. Overall treatment effects were also detected for $\%$ time in the open arm $\left(\mathrm{F}_{(1,55)}=5.9, P<0.05\right.$; Figure $\left.2 \mathrm{a}\right)$ and $\%$ entries in the open arms $\left(\mathrm{F}_{(1,55)}=7.4, P<0.01\right.$; Figure $\left.2 \mathrm{~b}\right)$ with rats treated with the NK1R antagonist spending more time and making more entries into the open arms compared with vehicle-treated rats. No significant effects were detected for entries into the closed arms, a measure of locomotor activity (Figure 2c).

Taken together, these results indicate that ShA and (to a greater degree) LgA to heroin self-administration increases anxiety-like behavior on the elevated plus maze. The findings also demonstrate an anxiolytic effect of L822429, as shown previously. However, there does not appear to be any difference in response to NK1R antagonism between ShA and LgA rats. Finally, the data indicate that the effects of heroin self-administration and drug treatment that were observed are not confounded by effects on locomotor activity.

\section{NK1R Antagonism Did Not Affect Mechanical Sensitivity in ShA or LgA Rats}

The results for PWT measurements (an indicator of mechanical sensitivity) are shown in Figure 3. Two-way ANOVA revealed a significant group effect $\left(\mathrm{F}_{(2,20)}=33.4\right.$, $P<0.0001)$. Post hoc testing showed that ShA rats displayed lower PWTs compared with naïve rats $(P<0.0001)$ and that LgA rats displayed lower PWTs compared with both ShA and naïve rats $(P<0.001)$. However, L822429 $(30 \mathrm{mg} / \mathrm{kg}) \mathrm{did}$ not affect mechanical sensitivity in any group.

\section{TacR1 mRNA Expression Levels are Changed in LgA and ShA Rats}

TacR1 mRNA levels in the PFC, NAc, BNST, AMYG, and Hipp of rats exposed repeatedly to moderate or escalating doses of heroin in a response-contingent or -non-contingent manner are shown in Figure 4.

Figure $4 \mathrm{a}$ shows the responding for heroin self-administration of the rats used for mRNA analysis. As expected, ShA rats displayed stable heroin intake, whereas LgA rats displayed escalation of heroin intake (group $\times$ session interaction: $\left.\mathrm{F}_{(12,132)}=4.6, P<0.0001\right)$ that became significant from session 5 onward $(P<0.05)$. There was a main group effect on TacR1 mRNA levels in the PFC $\left(\mathrm{F}_{(2,21)}=5.2\right.$, $P<0.05)$, NAc $\quad\left(\mathrm{F}_{(2,21)}=4.0, \quad P<0.05\right)$, and BNST $\left(\mathrm{F}_{(2,21)}=5.4, P<0.05\right)$. Post hoc testing revealed that ShA rats displayed lower TacR1 mRNA levels in the PFC $(P<0.05)$, NAc $(P<0.01)$, and BNST $(P<0.05)$ compared with naïve rats. LgA rats displayed lower TacR1 mRNA levels in the PFC and the Hipp compared with naïve rats $(P<0.05)$. A trend toward a reduction in TacR 1 was also observed in the NAc and BNST of LgA rats. ShA and LgA rats did not differ in expression of TacR 1 in the brain areas studied (Figure $4 \mathrm{~b}$ ).

To determine whether the observed changes in TacR 1 gene expression following heroin self-administration were 

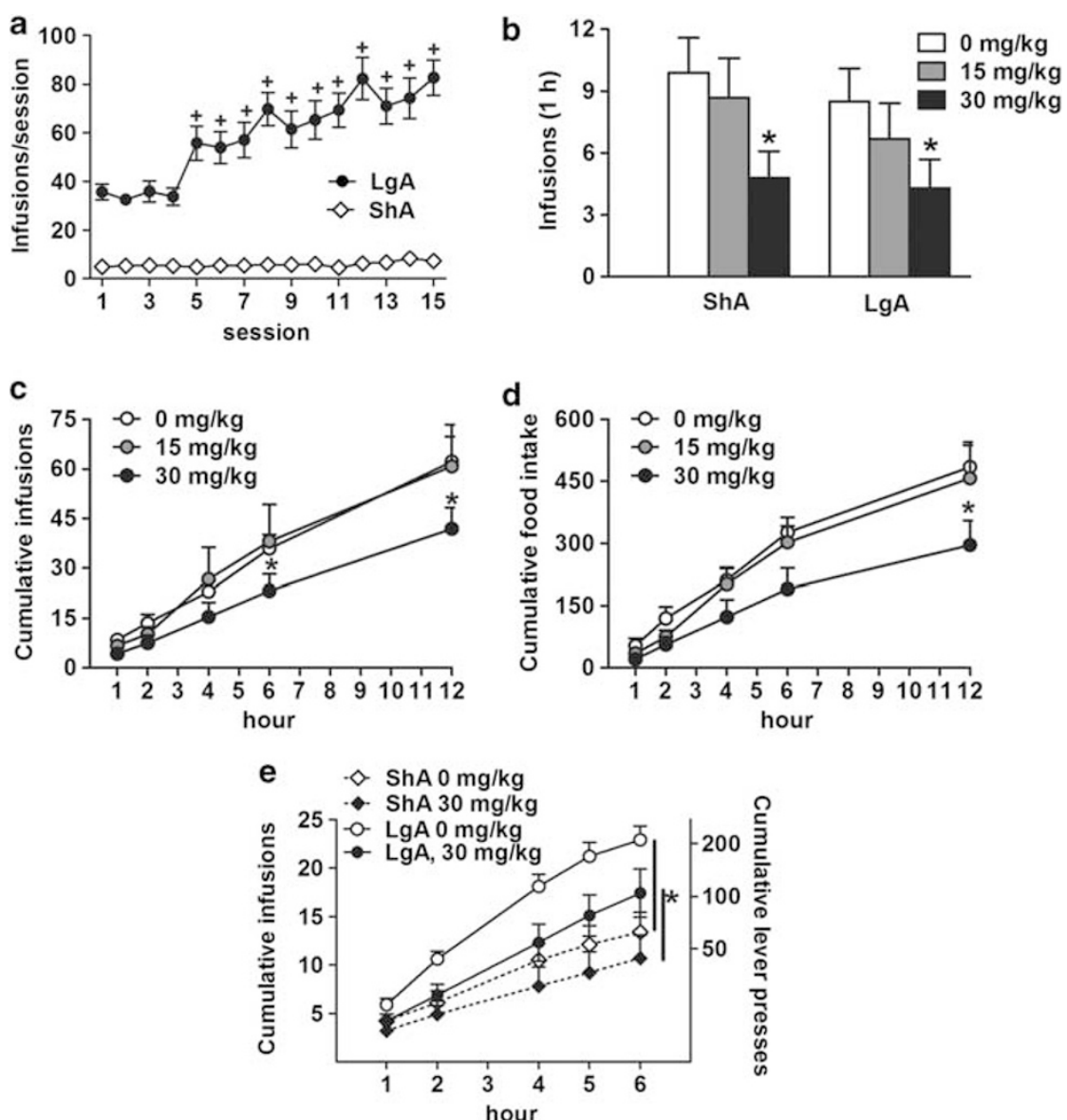

Figure I L822429 decreases heroin intake and motivation to consume heroin in both short access (ShA) and long access ( $L g A)$ rats. The bars or dots and vertical lines represent mean values and SEM. (a) Extended periods of drug access result in significantly increased heroin intake over time. (b) L822429 $(30 \mathrm{mg} / \mathrm{kg}$ ) significantly decreases responding (fixed-ratio I (FRI)) for heroin in the ShA and LgA rats during the first hour of heroin intake. (c) L822429 $(30 \mathrm{mg} / \mathrm{kg}$ ) significantly decreases responding (FRI) for heroin in the LgA rats in the entire I2-h session. (d) L822429 (30 mg/kg) significantly decreases responding (FR3) for food self-administration in the ShA and LgA rats. (e) L822429 (30 mg/kg) significantly decreases responding for heroin on a PR schedule of reinforcement in the ShA and LgA rats. ${ }^{*} P<0.05$ vehicle vs $L 822429 ;{ }^{+} P<0.05$ different from first session. $N=10$ per group.

Table I Total Session Responding for Food and Water

\begin{tabular}{lccc}
\hline Group & $\begin{array}{c}\text { L822429 } \\
\text { dose }(\mathbf{m g} / \mathbf{k g})\end{array}$ & $\begin{array}{c}\text { Food pellets } \\
\text { (nose pokes) }\end{array}$ & $\begin{array}{c}\text { Water } \\
\text { (nose pokes) }\end{array}$ \\
\hline ShA & 0 & $156 \pm 35$ & $22 \pm 8$ \\
& 15 & $120 \pm 34$ & $20 \pm 7$ \\
& 30 & $64 \pm 27 *$ & $17 \pm 5$ \\
LgA & 0 & $485 \pm 63$ & $343 \pm 51$ \\
& 15 & $457 \pm 84$ & $355 \pm 78$ \\
& 30 & $295 \pm 62 *$ & $384 \pm 55$ \\
\hline
\end{tabular}

$* P<0.05$, vs control; $N=10$ per dose. All values represent mean \pm SEM.

response-related or could be produced via passive, noncontingent heroin exposure, we chronically treated rats with heroin for 18 days, either at a constant level (repeated heroin group: $1.25 \mathrm{mg} / \mathrm{kg}$ subcutaneously, days $1-18$ ) or via an escalating dose regimen (escalated heroin group: $1.25-20 \mathrm{mg} / \mathrm{kg}$ subcutaneously), and compared gene expression changes relative to animals receiving chronic saline (repeated saline group). To ensure that this procedure produced heroin dependence, we tested their mechanical sensitivity using von Frey's filaments on day 18 just before the final injection (Figure 4c). There was a significant heroin treatment effect on mechanical sensitivity thresholds $\quad\left(\mathrm{F}_{(2,21)}=17.1, \quad P<0.0001\right)$. Repeated heroin injections significantly increased mechanical sensitivity compared with repeated saline injections $(P<0.01)$, whereas injections of escalating doses of heroin produced increased mechanical sensitivity compared with both repeated saline and repeated heroin injections $(P<0.05)$.

Figure $4 \mathrm{~d}$ shows TacR $1 \mathrm{mRNA}$ levels following passive subcutaneous injections of heroin either at a constant level (repeated heroin group: $1.25 \mathrm{mg} / \mathrm{kg}$ subcutaneously, days 118 ) or via an escalating dose regimen (escalated heroin group: $1.25-20 \mathrm{mg} / \mathrm{kg}$ subcutaneously), and compared gene expression changes relative to animals receiving chronic saline (repeated saline group). There was a main group effect on TacR1 mRNA levels in the PFC $\left(\mathrm{F}_{(2,21)}=13.39\right.$; $P<0.001)$, NAc $\left(\mathrm{F}_{(2,21)}=7.90 ; \quad P=0.003\right)$, and Hipp $\left(\mathrm{F}_{(2,21)}=4.37 ; P<0.02\right)$. Post hoc analysis revealed that TacR 1 mRNA levels were significantly increased in the 

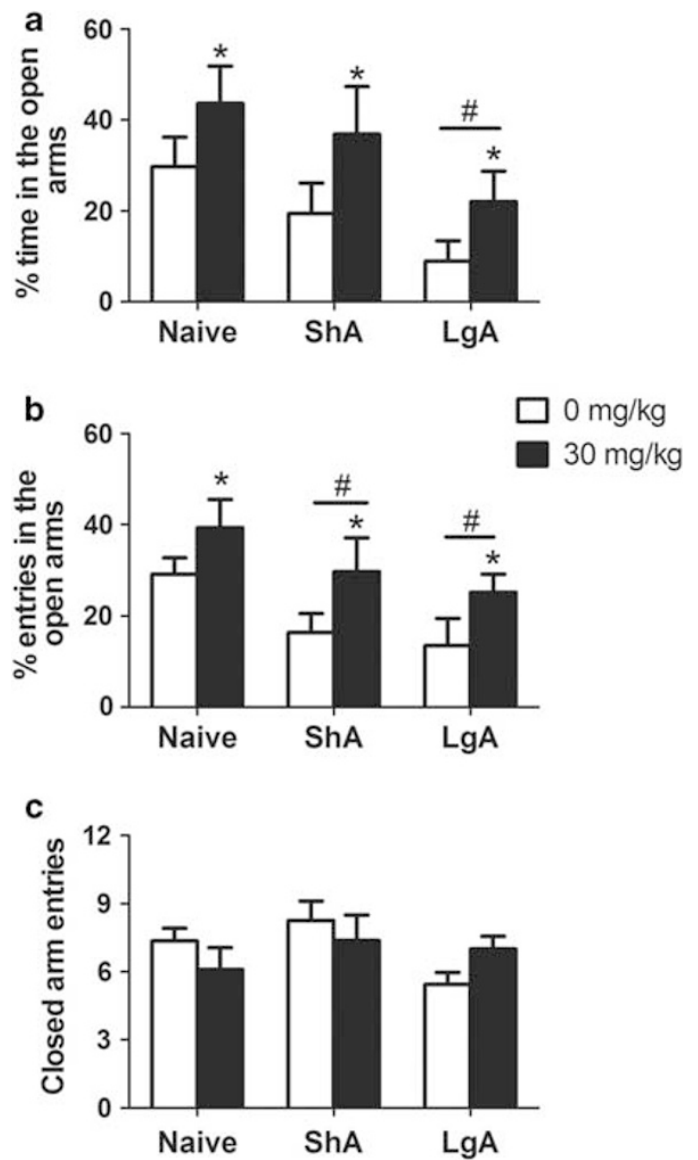

Figure 2 Anxiolytic-like effect of L822429 measured by the elevated plus maze. The bars and vertical lines represent mean values and SEM. (a) \% Time spent and (b) \% entries in the open arms. (c) Number of entries in the closed arms. $* P<0.05$ vehicle vs L822429; ${ }^{\#} P<0.05$ short access (ShA) or long access ( $\operatorname{Lg} A)$ rats vs naïve rats. $N=8-14$ per group.

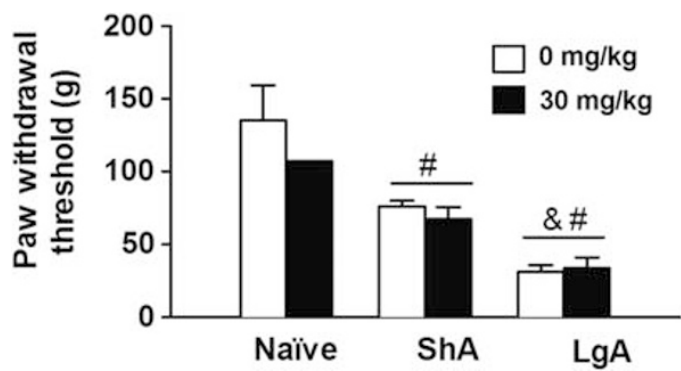

Figure 3 Paw withdrawal thresholds are significantly decreased in short access (ShA) rats, and to a significantly greater degree in long access ( $\operatorname{Lg} A)$ rats compared with naive rats. The bars and vertical lines represent mean values and SEM. L822429 (30 mg/kg) did not alter mechanical hypersensitivity in either ShA or $\operatorname{LgA}$ rats. ${ }^{\#} P<0.05$ ShA or $L g A$ rats vs naive rats; ${ }^{\&} p<0.05$ ShA vs LgA rats. $N=6$ to 7 per group.

mPFC of both repeated $(P<0.02)$ and escalated $(P<0.001)$ heroin groups compared with repeated saline group. Similarly, TacR1 mRNA levels were significantly increased in the NAc of the repeated heroin group compared with repeated saline group $(P<0.001)$. In addition, there was a significant difference in TacR 1 expression between repeated and escalated heroin groups. Levels of TacR1 mRNA in the mPFC are significantly higher in the escalated heroin group compared with the repeated heroin group $(P<0.02)$. In contrast, levels of TacR1 mRNA in the NAc and the Hipp were significantly lower in the escalated heroin group compared with the repeated heroin group $(P<0.02)$.

\section{DISCUSSION}

We report that the NK1R antagonist L822429 decreased both heroin self-administration and the motivation to obtain heroin, as measured by PR responding, with no apparent difference between ShA and LgA rats. The NK1R antagonist also produced an anxiolytic-like effect in ShA, $\operatorname{LgA}$, and naïve rats alike, whereas it did not alter the mechanical hypersensitivity that developed in animals selfadministering heroin. Consistent with the behavioral results, TacR 1 mRNA expression levels did not differ between ShA and LgA rats in stress/reward-related brain areas, but was significantly decreased in the PFC of both ShA and $\operatorname{LgA}$ rats compared with heroin-naïve rats. Similarly, TacR1 mRNA expression was significantly decreased in the NAc and BNST of ShA rats as well as in the Hipp of LgA rats. Furthermore, we observed a trend toward a reduction in the NAc and BNST of LgA rats. Interestingly, we also found that heroin-induced differences in TacR 1 mRNA expression may be due to the volitional consumption of heroin as opposed to the pharmacological effect of passive heroin exposure alone.

Our present findings are in line with prior studies on the role of the SP/NK1R system in opioid reward, but extend these in an important way. It has previously been shown that NK1R ${ }^{-1-}$ mice fail to develop CPP for morphine (Murtra et al, 2000) and self-administer lower levels of morphine than wild-type mice (Ripley et al, 2002). Because those data were obtained using constitutive knockout mice, they left unanswered a question that is critical to assess the potential of NK1R antagonists as a possible pharmacotherapy for opioid addiction: whether compensatory developmental effects contribute to the observed phenotype. Our present data indicate that reduction of heroin reinforcement by NK1R antagonism is not related to compensatory developmental mechanisms in the null mutants, but rather reflects a true pharmacological effect.

Our observations are consistent with the results of a recent study where pharmacological NK1R blockade attenuated the reward-potentiating effects of morphine, as measured by reversal of the morphine reduction in ICSS thresholds (Robinson et al, 2012). However, findings of reduced self-administration need to be interpreted with caution because they may be confounded by sedative or otherwise performance-impairing drug effects. In the present case, we believe that several observations make this unlikely. In this study, we show that the $30 \mathrm{mg} / \mathrm{kg} \mathrm{L} 822429$ dose did not influence the total number of entries into the closed arms of the elevated plus maze, a measure of locomotor activity. Furthermore, nose-poke activity for water, measured during heroin self-administration remained unchanged due to NK1R antagonism. Supporting the hypothesis of a specific effect of L822429 on heroin 
a

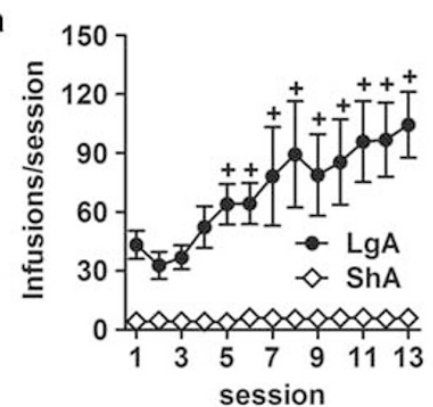

C

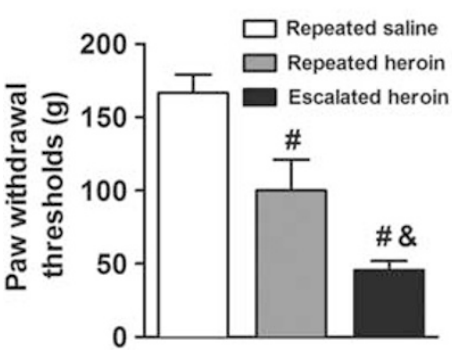

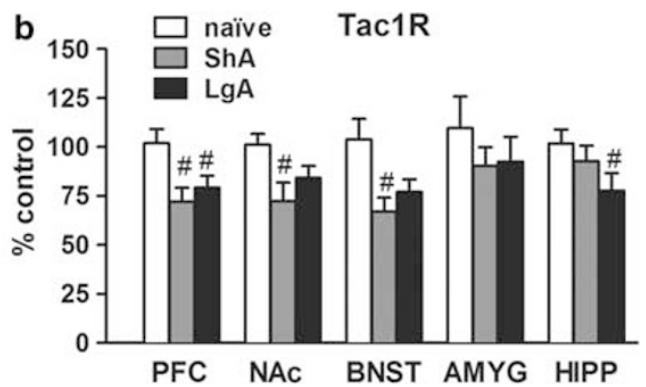

d

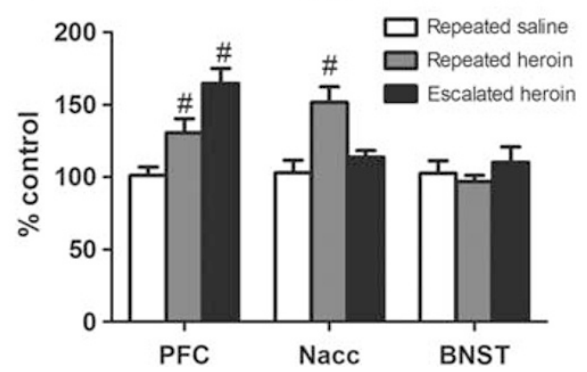

Figure 4 Expression of TacRI in stress/reward-related brain areas are presented as the percentage of control (naïve) values. The bars or dots and vertical lines represent mean values and SEM. (a) Heroin self-administration by short access (ShA) and long access (LgA) rats used for mRNA expression measurements. (b) TacR I mRNA levels in stress/reward-related brain areas of ShA, LgA, and naïve rats. (c) Mechanical sensitivity of rats repeatedly injected with saline, heroin, or escalated doses of heroin. (d) TacR I mRNA levels in stress/reward-related brain areas of rats repeatedly injected with saline, heroin, or escalated doses of heroin. ${ }^{\#} P<0.05$ ShA or LgA rats vs naïve rats and repeated heroin or escalated heroin vs repeated saline, \& $P<0.05$ repeated heroin vs escalated heroin, and ${ }^{+} P<0.05$ different from first session. $N=6-8$ per group.

self-administration, we have previously shown that $30 \mathrm{mg} /$ $\mathrm{kg}$ L822429 did not influence locomotor activity, alcohol, or sucrose self-administration in Wistar rats (Schank et al, 2011). However, we found here that NK1 antagonism does inhibit responding for food at the highest dose of L822429 tested. Because the food pellets available during selfadministration sessions are more palatable than their regular chow, the suppression of responding for food following L822429 treatment might be a direct effect of the drug on suppressing food reward, which is at least partially mediated by opioids (Barbano and Cador, 2006). Finally, the ability of L822429 to attenuate PR responding for heroin, which was most evident later on the testing session when the workload was higher, suggests that its effects on selfadministration are related to a specific motivational effect, a reduction of heroin reinforcement.

We found that L822429 suppressed heroin self-administration and motivation to obtain heroin in a similar manner in both ShA and LgA rats. This indicates that the SP/NK1R system does not contribute to the escalation of heroin selfadministration induced by extended drug access. Escalation of heroin intake is thought to reflect an important aspect of the transition from initial drug use to addiction (Ahmed and Koob, 2005) and has been associated with brain reward dysfunction. Rats that exhibit stable heroin self-administration display lowered ICSS thresholds upon heroin administration, whereas rats with extended access show a progressive increase in ICSS thresholds during escalation, indicating a tolerance to the rewarding effect of heroin (Kenny et al, 2006). In addition, escalation of heroin selfadministration in LgA rats produces a parallel development of mechanical hypersensitivity (Edwards et al, 2012) and anxiogenic-like effects (present results), suggesting that a recruitment of negative emotional states such as pain and anxiety may, in part, drive excessive heroin intake. Accordingly, previous studies found an increase in stressinduced reinstatement to heroin-seeking behavior in LgA compared with ShA rats (Ahmed et al, 2000).

Given the established role of the SP/NK1R system in anxiety-like behavior (reviewed by Ebner and Singewald (2006)), we investigated whether this system might contribute to heroin withdrawal-induced negative emotional states as modeled in $\operatorname{LgA}$ rats. We found an anxiolytic-like effect of L822429, indicated by an increased exploration of the open arms of the elevated plus maze. This result is consistent with several preclinical studies showing that genetic or pharmacological NK1R inactivation decreases anxietylike behavior (reviewed by Ebner and Singewald (2006)). However, L822429 displayed similar anxiolytic-like effects in both ShA and LgA rats. Furthermore, L822429 did not affect mechanical hypersensitivity (a model of hyperalgesia) observed in heroin self-administering rats. Hyperalgesia has been proposed as another component of negative emotional states that may drive the transition to opioid addiction (Shurman et al, 2010). Taken together, these observations argue against a specific role for the SP/NK1R system in the recruitment of negative emotionality that contributes to the escalation of heroin self-administration, although the SP/ NK1R system may be engaged early on in the neuroadaptations associated with initial opioid self-administration.

Further support for this conclusion comes from our gene expression analysis, in which ShA and $\operatorname{LgA}$ heroin 
self-administration resulted in similar TacR1 expression profiles. We found a decrease in TacR1 mRNA levels in the PFC of both ShA and LgA rats compared with heroin-naïve rats. A trend toward a reduction in TacR1 was also observed in the NAc and BNST. It has been suggested that administration of opioids elicits SP release (Commons, 2010). It has also been shown that SP release causes an increase in NK1R internalization (Kramer et al, 1998). In our study, TacR1 mRNA levels were measured during heroin withdrawal. Thus, although we cannot exclude the possibility that the reduction in TacR1 mRNA does not reflect a reduction in protein, the decrease of TacR1 mRNA may reflect complementary changes in NK1R expression, potentially driven by NK1R activation and internalization.

Furthermore, our gene expression results suggest that heroin-induced changes in TacR1 mRNA expression are not only dependent on the pharmacological effects of heroin but appear to be heavily dependent on response contingency. These results are in line with previous studies demonstrating differential neuroadaptations in rats receiving heroin in a response-contingent $v s$-non-contingent manner (Kuntz et al, 2008; Jacobs et al, 2004; Jacobs et al, 2005), and these data may have particular importance for addiction and pain management fields, respectively. Interestingly, we also found that increases in NAc TacR1 mRNA following repeated (moderate dose) heroin was reduced following non-contingent, escalated-dose heroin exposure, possibly reflecting tolerance-associated changes in the NK1 receptor system. This finding is also in accordance with a previous study implicating the NAc in tolerance-related neuroadaptations in relation to chronic cocaine exposure (Edwards et al, 2010). Other factors may be in play regarding the differential effect of passive $v s$ operant administration of heroin on TacR1 expression levels. Indeed, passive administration was performed via subcutaneous injections, whereas the heroin was infused intravenously in the self-administration experiments. Therefore, different pharmacokinetics may contribute to the opposite effects of passive $v s$ operant heroin administration on TacR1 mRNA expression.

In conclusion, we have demonstrated that pharmacological NK1R blockade reduces heroin self-administration in rats. This is likely the result of its ability to attenuate acute, positively reinforcing properties of heroin. In contrast, our findings do not provide support for a specific role of the SP/NK1R system in heroin intake escalation or potentiation of negative emotional states that may drive the negative reinforcing properties of heroin. However, these data suggest that NK1R antagonists may have potential as pharmacotherapies for specific components of the opioid dependence cycle. Maintenance treatment of heroin addiction with the opioid agonist methadone or the partial agonist buprenorphine is highly effective (Amato et al, 2005), but other effective treatments are largely lacking for early-stage opioid dependence when replacement therapy may not be justified. NK1R antagonists are safe and well tolerated in humans, including alcoholics (Kramer et al, 1998, 2004; ; Furmark et al, 2005; George et al, 2008). Taken together, we believe that these observations provide a rationale for exploring the potential of NK1R antagonism as a first-line treatment in early-stage opioid dependence.

\section{ACKNOWLEDGEMENTS}

We thank Dr Eric Augier for its insightful comments regarding the manuscript. This research was supported by the intramural program of the National Institute on Alcohol Abuse and Alcoholism (MH) and the National Institute on Drug Abuse (KR). This work was also supported by grants from the National Institute on Drug Abuse (DA004043, GFK), a Research Career Scientist Award from the Biomedical Laboratory Research and Development Program, Veterans Health Administration (GS), and by the Pearson Center for Alcoholism and Addiction Research. This is article number 21813 from the Scripps Research Institute.

\section{DISCLOSURE}

The authors declare that, except for income received from our primary employer, no financial support or compensation has been received from any individual or corporate entity over the past 3 years for research or professional service and there are no personal financial holdings that could be perceived as constituting a potential conflict of interest.

\section{REFERENCES}

Ahmed SH, Koob GF (2005). Transition to drug addiction: a negative reinforcement model based on an allostatic decrease in reward function. Psychopharmacology 180: 473-490.

Ahmed SH, Walker JR, Koob GF (2000). Persistent increase in the motivation to take heroin in rats with a history of drug escalation. Neuropsychopharmacology 22: 413-421.

Amato L, Davoli M, Perucci A, Ferri M, Faggiano F, Mattick P (2005). An overview of systematic reviews of the effectiveness of opiate maintenance therapies: available evidence to inform clinical practice and research. J Subst Abuse Treat 28: 321-329.

Barbano MF, Cador M (2006). Differential regulation of the consummatory, motivational and anticipatory aspects of feeding behavior by dopaminergic and opioidergic drugs. Neuropsychopharmacology 31: 1371-1381.

Chaplan SR, Bach FW, Pogrel JW, Chung JM, Yaksh TL (1994). Quantitative assessment of tactile allodynia in the rat paw. J Neurosci Methods 53: 55-63.

Commons KG (2010). Neuronal pathways linking substance P to drug addiction and stress. Brain Res 1314: 175-182.

Dixon WJ (1980). Efficient analysis of experimental observations. Annu Rev Pharmacol Toxicol 20: 441-462.

Ebner K, Muigg P, Singewald G, Singewald N (2008). Substance P in stress and anxiety: NK-1 receptor antagonism interacts with key brain areas of the stress circuitry. Ann N Y Acad Sci 1144: 61-73.

Ebner K, Rupniak NM, Saria A, Singewald N (2004). Substance P in the medial amygdala: emotional stress-sensitive release and modulation of anxiety-related behavior in rats. Proc Natl Acad Sci USA 101: 4280-4285.

Ebner K, Singewald N (2006). The role of substance P in stress and anxiety responses. Amino Acids 31: 251-272.

Edwards S, Koob GF (2010). Neurobiology of dysregulated motivational systems in drug addiction. Fut Neurol 5: 393-401.

Edwards S, Vendruscolo LF, Schlosburg JE, Misra KK, Wee S, Park PE et al (2012). Development of mechanical hypersensitivity in rats during heroin and ethanol dependence: alleviation by CRF receptor antagonism. Neuropharmacology 62: 1142-1151. 
Furmark T, Appel L, Michelgard A, Wahlstedt K, Ahs F, Zancan S et al (2005). Cerebral blood flow changes after treatment of social phobia with the neurokinin-1 antagonist GR205171, citalopram, or placebo. Biol Psychiatry 58: 132-142.

Gadd CA, Murtra P, De Felipe C, Hunt SP (2003). Neurokinin-1 receptor-expressing neurons in the amygdala modulate morphine reward and anxiety behaviors in the mouse. J Neurosci 23: 8271-8280.

George DT, Gilman J, Hersh J, Thorsell A, Herion D, Geyer C et al (2008). Neurokinin 1 receptor antagonism as a possible therapy for alcoholism. Science 319: 1536-1539.

Jacobs EH, de Vries TJ, Smit AB, Schoffelmeer AN (2004). Gene transcripts selectively down-regulated in the shell of the nucleus accumbens long after heroin self-administration are up-regulated in the core independent of response contingency. FASEB $J$ 18: 200-202.

Jacobs EH, Smit AB, de Vries TJ, Schoffelmeer AN (2005). Longterm gene expression in the nucleus accumbens following heroin administration is subregion-specific and depends on the nature of drug administration. Addict Biol 10: 91-100.

Kenny PJ, Chen SA, Kitamura O, Markou A, Koob GF (2006). Conditioned withdrawal drives heroin consumption and decreases reward sensitivity. J Neurosci 26: 5894-5900.

Kramer MS, Cutler N, Feighner J, Shrivastava R, Carman J, Sramek JJ et al (1998). Distinct mechanism for antidepressant activity by blockade of central substance $\mathrm{P}$ receptors. Science 281: 1640-1645.

Kramer MS, Winokur A, Kelsey J, Preskorn SH, Rothschild AJ, Snavely D et al (2004). Demonstration of the efficacy and safety of a novel substance $\mathrm{P}$ (NK1) receptor antagonist in major depression. Neuropsychopharmacology 29: 385-392.

Kuntz KL, Patel KM, Grigson PS, Freeman WM, Vrana KE (2008). Heroin self-administration: II. CNS gene expression following withdrawal and cue-induced drug-seeking behavior. Pharmacol Biochem Behav 90: 349-356.

Leffler A, Ahlstedt I, Engberg S, Svensson A, Billger M, Oberg L et al (2009). Characterization of species-related differences in the pharmacology of tachykinin NK receptors 1, 2 and 3. Biochem Pharmacol 77: 1522-1530.
Murtra P, Sheasby AM, Hunt SP, De Felipe C (2000). Rewarding effects of opiates are absent in mice lacking the receptor for substance P. Nature 405: 180-183.

Ripley TL, Gadd CA, De Felipe C, Hunt SP, Stephens DN (2002). Lack of self-administration and behavioural sensitisation to morphine, but not cocaine, in mice lacking NK1 receptors. Neuropharmacology 43: 1258-1268.

Ririe DG, Eisenach JC (2006). Age-dependent responses to nerve injury-induced mechanical allodynia. Anesthesiology 104: 344-350.

Robinson JE, Fish EW, Krouse MC, Thorsell A, Heilig M, Malanga CJ (2012). Potentiation of brain stimulation reward by morphine: effects of neurokinin-1 receptor antagonism. Psychopharmacology (Berl) 220: 215-224.

Schank JR, Pickens CL, Rowe KE, Cheng K, Thorsell A, Rice KC et al (2011). Stress-induced reinstatement of alcohol-seeking in rats is selectively suppressed by the neurokinin 1 (NK1) antagonist L822429. Psychopharmacology (Berl) 218: 111-119.

Shurman J, Koob GF, Gutstein HB (2010). Opioids, pain, the brain, and hyperkatifeia: a framework for the rational use of opioids for pain. Pain Med 11: 1092-1098.

Singewald N, Chicchi GG, Thurner CC, Tsao KL, Spetea M, Schmidhammer $\mathrm{H}$ et al (2008). Modulation of basal and stressinduced amygdaloid substance $\mathrm{P}$ release by the potent and selective NK1 receptor antagonist L-822429. J Neurochem 106: 2476-2488.

Thorsell A, Schank JR, Singley E, Hunt SP, Heilig M (2010). Neurokinin-1 receptors (NK1R:s), alcohol consumption, and alcohol reward in mice. Psychopharmacology (Berl) 209: 103-111.

Vendruscolo LF, Barbier E, Schlosburg JE, Misra KK, Whitfield TW Jr., Logrip ML et al (2012). Corticosteroid-dependent plasticity mediates compulsive alcohol drinking in rats. J Neurosci 32: 7563-7571.

Vendruscolo LF, Schlosburg JE, Misra KK, Chen SA, Greenwell TN, Koob GF (2011). Escalation patterns of varying periods of heroin access. Pharmacol Biochem Behav 98: 570-574.

Vendruscolo LF, Takahashi RN, Bruske GR, Ramos A (2003). Evaluation of the anxiolytic-like effect of NKP608, a NK1receptor antagonist, in two rat strains that differ in anxietyrelated behaviors. Psychopharmacology (Berl) 170: 287-293. 